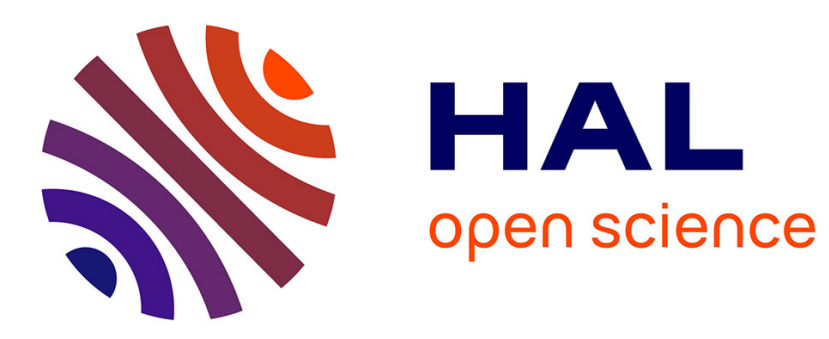

\title{
Dephasing processes in a single semiconductor quantum dot
}

\author{
Guillaume Cassabois, Robson Ferreira
}

\section{To cite this version:}

Guillaume Cassabois, Robson Ferreira. Dephasing processes in a single semiconductor quantum dot. Comptes Rendus. Physique, 2008, 9 (8), pp.830. hal-00465011

\section{HAL Id: hal-00465011 https://hal.science/hal-00465011}

Submitted on 18 Mar 2010

HAL is a multi-disciplinary open access archive for the deposit and dissemination of scientific research documents, whether they are published or not. The documents may come from teaching and research institutions in France or abroad, or from public or private research centers.
L'archive ouverte pluridisciplinaire HAL, est destinée au dépôt et à la diffusion de documents scientifiques de niveau recherche, publiés ou non, émanant des établissements d'enseignement et de recherche français ou étrangers, des laboratoires publics ou privés. 


\title{
Dephasing processes in a single semiconductor quantum dot
}

\author{
Guillaume Cassabois ${ }^{\mathrm{a}}$, Robson Ferreira ${ }^{\mathrm{a}}$ \\ ${ }^{a}$ Ecole Normale Supérieure, Laboratoire Pierre Aigrain, 24, rue Lhomond 75231 Paris Cedex 5, France \\ CNRS UMR8551, Laboratoire associé aux universités Pierre et Marie Curie et Paris Diderot, France \\ Received $* * * * * ;$ accepted after revision +++++
}

\begin{abstract}
We discuss the decoherence dynamics in a single semiconductor quantum dot and analyze two dephasing mechanisms. In the first part of the review, we examine the intrinsic source of dephasing provided by the coupling to acoustic phonons. We show that the non-perturbative reaction of the lattice to the interband optical transition results in a composite optical spectrum with a central zero-phonon line and lateral side-bands. In fact, these acoustic phonon side-bands completely dominate the quantum dot optical response at room temperature. In the second part of the paper, we focus on the extrinsic dephasing mechanism of spectral diffusion that determines the quantum dot decoherence at low temperatures. We interpret the variations of both width and shape of the zerophonon line as due to the fluctuating electrostatic environment. In particular, we demonstrate the existence of a motional narrowing regime in the limit of low incident power or low temperature, thus revealing an unconventional phenomenology compared to nuclear magnetic resonance.
\end{abstract}

\section{Résumé}

Processus de déphasage dans une boîte quantique unique de semiconducteurs. Nous discutons la dynamique de décohérence pour une boîte quantique unique et analysons deux mécanismes de déphasage. Dans la première partie de cette revue, nous examinons la source intrinsèque de déphasage provenant du couplage aux phonons acoustiques. Nous montrons que la réaction non-perturbative du réseau à la transition optique interbande se traduit par un spectre optique composite avec une raie centrale à zéro-phonon et des bandes latérales. De fait, les bandes latérales de phonons acoustiques dominent complètement la réponse optique de la boîte quantique à température ambiante. Dans la deuxième partie de cet article, nous nous concentrons sur le mécanisme extrinsèque de déphasage associé à la diffusion spectrale, qui détermine le spectre de la boîte quantique à basses températures. Nous interprétons les variations de profil et de largeur de la raie à zéro-phonon comme étant dues à l'environnement électrostatique fluctuant. Nous montrons en particulier l'existence d'un régime de rétrécissement par le mouvement dans la limite des basses températures ou faibles densités d'excitation, ce qui révèle une phénoménologie non-conventionnelle par rapport à la résonance magnétique nucléaire.

Key words: decoherence; acoustic phonon; spectral diffusion; motional narrowing

Mots-clés : décohérence; phonon acoustique; diffusion spectrale; rétrécissement par le mouvement

Email addresses: guillaume.cassabois@lpa.ens.fr (Guillaume Cassabois), robson.ferreira@lpa.ens.fr (Robson Ferreira). 


\section{Introduction}

One leitmotif of contemporary physics is the search for ideal systems displaying coherent optical response. In this context, a strong effort has been put on the study of the optical response of nanostructures built up on semiconductor matrix. This is a non-trivial issue, since it is widely admitted that dephasing processes are ubiquitous in semiconductors. In bulk this stems from two main effects : the existence of continua of states for electrons and the fact that an actual solid is not perfectly periodic but display defects. Thus, dephasing results from the fact that propagating electrons can undergo scatterings among states that are differently coupled to light. Ought decreasing dephasing by only increasing the crystal quality is hopeless, since residual static defects are always present despite the great advances in growth and, most importantly, because of the unavoidable thermal vibrations of the crystal lattice. A more promising route is to suppress carriers motion. This has strongly motivated the research in quantum dots (QDs) : carriers become localised in space in a nanometric region, while their low-lying electronic spectrum become a discrete sequence of levels. Recent decisive progresses in high-resolution local-probe optical spectroscopy have actually allowed showing that the optical spectra (luminescence, absorption) of a single QD display thin lines, reinforcing thereby the widespread image of single QDs as macro-atoms. This has brought a new paradigm for future devices : such atoms are at rest (no motion-related broadenings) in a solid matrix (attractive for manufacturing purposes). Subsequent studies have nevertheless allowed demonstrating very important departures from the ideal atomic-like situation of Lorentzian lines with widths limited by the sole radiative coupling. Non-lorentzian lineshapes and/or additional broadenings are currently observed in many QDs. These have been related to different sources : non-perturbative coupling to phonons and non-trivial relationships between the QD and its near environment. The first one is intrinsic to a crystal lattice whereas the second one depends on the precise experimental conditions and can thus to a certain extent be minimized. We focus in this review on these two couplings, which affect the QD electronic states and ultimately limit their coherent optical monitoring.

\section{Acoustic phonon dephasing}

The electron-phonon interaction provides one of the most efficient pathway for population and coherence relaxation in semiconductor nanostructures. In bulk materials, quantum wells or quantum wires, the translational invariance respectively in three, two or one direction leads to the existence of a continuum of states, whose density depends on the system dimensionality. Consequently, the electron-phonon interaction is conveniently described in the framework of the Fermi golden rule where the emission or the absorption of acoustic and optical phonons contributes to the dephasing rate with an efficiency given by the corresponding density of electronic states of the system. In semiconductor QDs, the three-dimensional quantum confinement leads to a full discretization of the electron and hole states that thus asks for a complete reexamination of the standard picture based on the application of the Fermi golden rule in the weak coupling regime. This theoretical issue is motivated by the experimental evidence of a non-Lorentzian optical spectrum in semiconductor QDs fabricated in various materials.

\subsection{Non-lorentzian optical spectrum}

The deviation from a Lorentzian profile was first reported by Besombes et al. in a single II-VI CdTe/ZnTe self-assembled QD [1]. As shown in Fig. 1, the photoluminescence (PL) spectrum displays a composite shape with a Lorentzian central part (fitted by the solid line) and lateral sidebands. Moreover, at low temperature (30K in Fig. 1), the sidebands appear to be asymmetric with a higher PL intensity for the low energy component. This deviation from a Lorentzian profile is not specific to II-VI QDs and it was later observed by single QD spectroscopy in III-V QDs in the InAs/GaAs system [2], or GaAs/AlAs one [3]. Time-resolved four-wave mixing experiments also gave a hint for the presence of lateral sidebands, but without a detailed insight into the relative intensity of the low and high energy sidebands [4].

The asymmetry of the sidebands becomes less and less pronounced on increasing the temperature, as shown in Fig. 2(a) where the redshift of the PL spectra with temperature follows the temperature variation of the band-gap energy of InAs. Above 70K the lateral sidebands are symmetric and tend to completely dominate the QD line [2]. In order to quantify the deviation from a Lorentzian profile, the ZPL fraction can be derived by calculating the ratio between the spectrally integrated intensities of the Lorentzian fit (solid line in Fig. 2(a)) and the experimental 


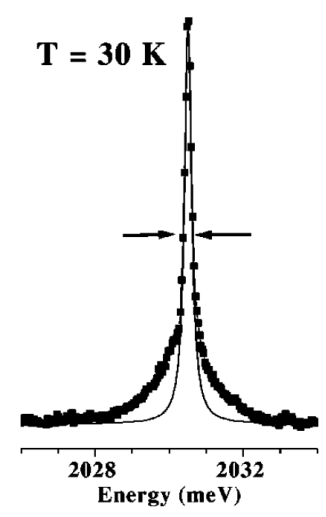

Figure 1. From Ref. [1]. Deviation from a Lorentzian emission spectrum. Photoluminescence spectrum of a single CdTe/ZnTe quantum dot at $30 \mathrm{~K}$, compared to a Lorentzian fit (solid line) adjusted to the central part of the spectrum.

data. As shown in the inset of this figure, the ZPL fraction decreases from $80 \%$ at low temperature to negligible values around $80 \mathrm{~K}$ when the $\mathrm{PL}$ spectrum is entirely given by the lateral sidebands. As a matter of fact, the homogeneous linewidth of the QD optical spectrum at room temperature is determined by the sidebands spectral extension [5]. We will see below that the lateral sidebands at lower (higher) energy are due to the recombination of one electron-hole pair with the net emission (absorption) of several acoustic phonons.

\subsection{Acoustic phonon sidebands}

The theoretical description of the lateral sidebands is an extension to electron-hole pairs of the Huang-Rhys formalism developed in the context of electron-phonon interaction in localized electronic states [6]. This model is based on the exact diagonalization of the hamiltonian of a system composed of a discrete electron state coupled to a continuum of acoustic phonons. In our case where an electron-hole pair is confined in a semiconductor QD, such a hamiltonian reads:

$$
H=E_{0}+\sum_{\vec{q}} \hbar \omega_{\vec{q}} c_{\vec{q}}^{+} c_{\vec{q}}+\sum_{\vec{q}} \hbar \omega_{\vec{q}}\left(f_{\vec{q}}^{*} c_{\vec{q}}^{+}+f_{\vec{q}} c_{\vec{q}}\right)
$$

where $E_{0}$ is the energy of the $\left|S_{e}, S_{h}\right\rangle$ state which is the first state of the QD hosting one bound electron-hole pair, $\hbar \omega_{\vec{q}}$ the energy of an acoustic phonon of wave-vector $\vec{q}, c_{\vec{q}}$ the annihilation operator of one phonon of wavevector $\vec{q}, f_{\vec{q}}$ gives the strength of the coupling to acoustic phonons and reads $\left\langle S_{e}, S_{h}, 0\left|V^{d}\right| S_{e}, S_{h}, 1_{\vec{q}}\right\rangle / \hbar \omega_{\vec{q}}$, where $V^{d}$ is the deformation potential interaction. By taking $a_{\vec{q}}=c_{\vec{q}}+f_{\vec{q}}^{*}$, the system hamiltonian then reads:

$$
H=E_{0}-\sum_{\vec{q}} \hbar \omega_{\vec{q}} f_{\vec{q}} f_{\vec{q}}^{*}+\sum_{\vec{q}} \hbar \omega_{\vec{q}} a_{\vec{q}}^{+} a_{\vec{q}}
$$

where the new annihilation operator $a_{\vec{q}}$ describes a bosonic quasi-particle since $\left[a_{\vec{q}}, a_{\vec{l}}^{+}\right]=\delta_{\vec{q}, \vec{l}}$. The hamiltonian of the system formed of the fundamental state $\left|S_{e}, S_{h}\right\rangle$ coupled to acoustic phonons is transformed into an hamiltonian of independent bosons, thus showing the need for taking into account the degrees of freedom of the phonon reservoir together with the electronic ones in a QD. This effect arises from the strong quantum confinement in the semiconductor QD where the local deformation of the crystal lattice and the presence of carriers in the nanostructure are spatially localized. The new fundamental state of the QD coupled to the acoustic phonon reservoir is thus given by:

$$
\begin{aligned}
\left|\widehat{S_{e}, S_{h}}\right\rangle & =\exp \left(-\sum_{\vec{q}} \hbar \omega_{\vec{q}} f_{\vec{q}} f_{\vec{q}}^{*} / 2\right) \exp \left(\sum_{\vec{q}} \hbar \omega_{\vec{q}} f_{\vec{q}}^{*} c_{\vec{q}}^{+}\right)\left|S_{e}, S_{h}, 0\right\rangle \\
& =\left|S_{e}, S_{h}\right\rangle \otimes \exp \left(-\sum_{\vec{q}} \hbar \omega_{\vec{q}} f_{\vec{q}} f_{\vec{q}}^{*} / 2\right) \exp \left(\sum_{\vec{q}} \hbar \omega_{\vec{q}} f_{\vec{q}}^{*} c_{\vec{q}}^{+}\right)|0\rangle
\end{aligned}
$$

where we see that the fundamental state $\left|\widehat{S_{e}, S_{h}}\right\rangle$ can be factorized in the form $\left|S_{e}, S_{h}\right\rangle \otimes\left|\Psi_{p h}\right\rangle$ where the $\left|\Psi_{p h}\right\rangle$ state describes the acoustic phonon reservoir. This means that the electron-acoustic phonon interaction is not in the strong coupling regime in a $\mathrm{QD}$ since there is no entanglement between the electron and phonon states. 

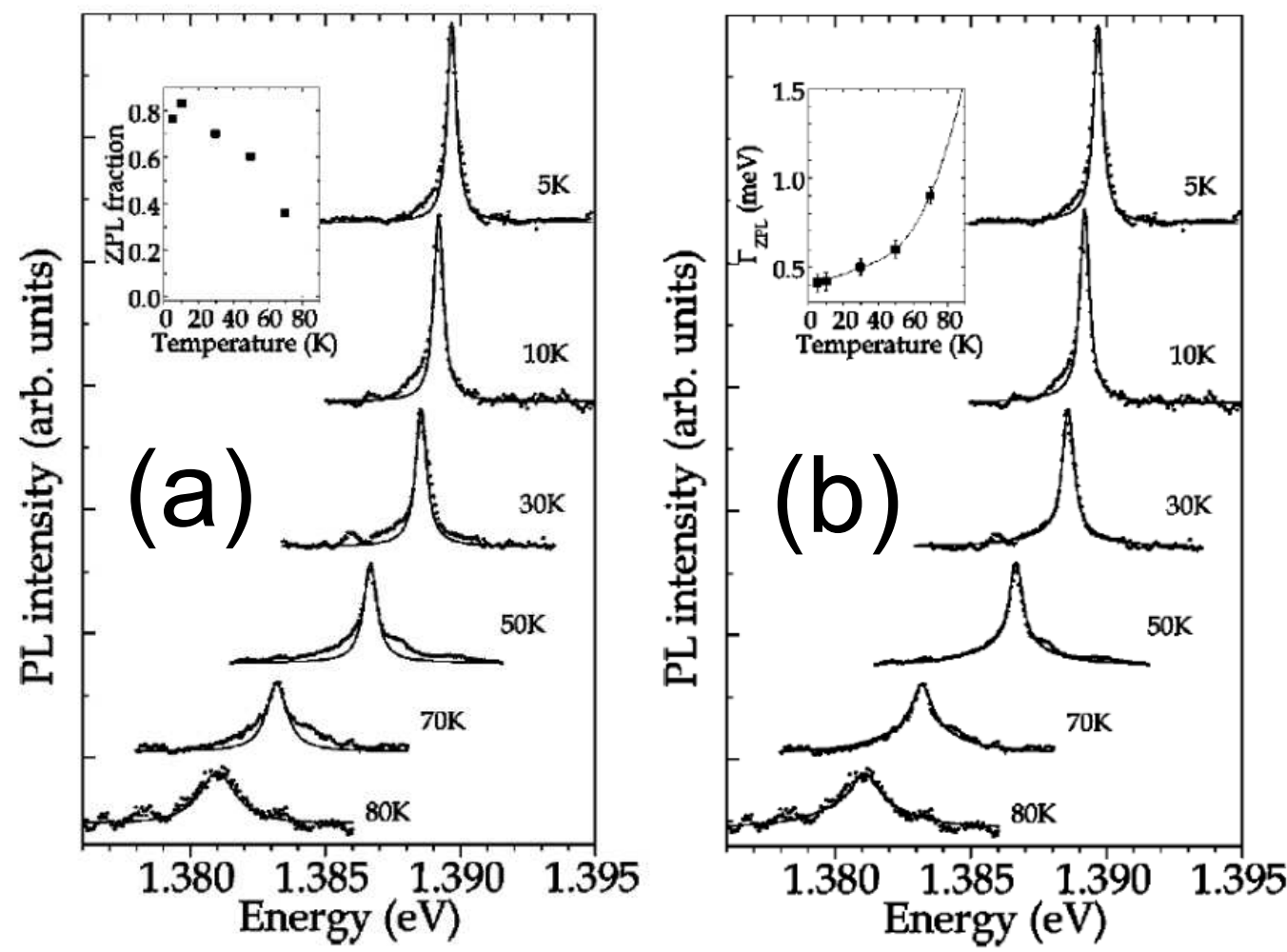

Figure 2. From Ref. [2]. Photoluminescence spectra of a single InAs/GaAs QD in the temperature range 5K-80K, normalized to the spectrally integrated intensity for each temperature. (a) Comparison with Lorentzian fits of the central part of the line. Inset: zero-phonon line fraction versus temperature. (b) Comparison with calculations in the framework of the Huang-Rhys model. Inset: temperature dependence of the zero-phonon linewidth deduced from the Lorentzian fits of (a).

However, it is worth to point out that the independent boson scheme allows an exact diagonalization of the electron-phonon coupling, and thus that the changing from bulk phonon modes for the empty QD towards $\left|\Psi_{p h}\right\rangle$ when the QD hosts one electron/hole pair represents a non-perturbative reaction of the lattice to the interband transition. As we recall below, this effect explains the existence of lateral sidebands in the QD PL spectrum and constitutes an intrinsic source of optical dephasing.

The optical spectrum of the fundamental transition is obtained by including the dipolar interaction and by calculating first the probability $W_{\overrightarrow{q_{i}}}\left(p_{i}\right)$ of having $p_{i}$ acoustic phonons of wave-vector $\overrightarrow{q_{i}}$ emitted $\left(p_{i}<0\right)$ or absorbed $\left(p_{i}>0\right)$ during the radiative recombination of the $\left|S_{e}, S_{h}\right\rangle$ state [6]. Finally, the spectral distribution of the emitted photons is obtained by summing over all possible configurations:

$$
I(E) \propto \sum_{\vec{p}} \prod_{i=1}^{N} W_{\overrightarrow{q_{i}}}\left(p_{i}\right) \delta\left(E-E_{0}-\sum_{j=1}^{N} p_{j} \hbar c_{s} q_{j}\right)
$$

where the quasi-continuum of acoustic phonons is described within the Debye approximation $\left(\omega_{\vec{q}}=c_{s} q\right)$ and is discretized into $N$ modes $(N \gg 1), \vec{p}=\left\{p_{1}, p_{2} \ldots p_{N}\right\}$ and each $p_{i}$ varies between $-\infty$ and $+\infty$.

The optical spectrum can be decomposed into a central so-called zero-phonon line (ZPL) related to the radiative recombination of the $\left|S_{e}, S_{h}\right\rangle$ without any net energy exchange with the phonons reservoir, that is $\sum_{j=1}^{N} p_{j} \hbar c_{s} q_{j}=0$ $(\vec{p}=\overrightarrow{0}$ corresponds the particular case where no phonon is absorbed or emitted). The sidebands of the ZPL come from the emission of photons at lower or higher energy depending on the sign of $\sum_{j=1}^{N} p_{j} \hbar c_{s} q_{j}$, respectively negative or positive. At that stage of the theoretical description, we face a fundamental limitation of our model since we predict a zero-phonon line with a zero width in contrast to the experimental data shown in Fig. 2 where the 
central Lorentzian part of the spectrum has a finite width of tens of $\mu \mathrm{eV}$. The finite width of the zero-phonon line can not be accounted for by our model based on the Huang-Rhys formalism with a first-order coupling to acoustic phonons. We will come back later to the essential issue of the ZPL width, but let us focus now on the sidebands. Indeed, we have to introduce phenomenologically the ZPL width. For that purpose, we convolute the optical spectrum given by Eq. (5) with a Lorentzian of width $\Gamma_{Z P L}$. In the calculated profiles (solid lines in Fig. 2(b)), we take for the input parameter $\Gamma_{Z P L}$ the values deduced from the Lorentzian fits adjusted to the central part of the experimental data (Fig. 2(a)). The excellent agreement between experimental data and theoretical fits on the whole temperature range demonstrates that the deviation from a Lorentzian line comes from the interaction with acoustic phonons that leads the appearance of lateral sidebands.

The spectral extent of the sidebands is mainly given by the mean energy of the acoustic phonons involved in the process. The wavelength of these phonons is of the order of the lateral size of the QD, thus giving a mean energy in the meV range. At low temperature $(k T \ll \mathrm{meV})$, the probability of absorbing phonons, which is proportional to the phonon occupation factor, is negligible and only the emission processes take place: the lateral sidebands are thus asymmetric with only the low energy component. At high temperature $(k T \gg m e V)$, absorption and emission get the same efficiency and the sidebands become symmetric. Finally, we would like to comment on the visibility of the lateral sidebands that depends on the ZPL width. Indeed, the convolution of the normalized Lorentzian with the calculated Huang-Rhys spectrum does not modify the intensity of the quasi-continuum associated to the broad lateral sidebands. On the contrary, the intensity maximum of the ZPL is inversely proportional to its linewidth. Consequently, the observation of the lateral sidebands tends to be easier for a broad ZPL because of the reduced intensity ratio between the central peak of the ZPL and the phonon sidebands. For a narrower ZPL, the deviation from a Lorentzian profile becomes more challenging and requires higher signal-to-noise ratios [2]. This means also that when looking for the narrowest QD line close to the radiative limit, the lateral sidebands are hardly observable.

Phonon sidebands bring an intrinsic source of dephasing for the optical transitions. Indeed, the dynamical counterpart of the broad sideband is a fast non-exponential decrease of the interband coherence at short times. Vagov and co-workers showed that part of the energy stored in the system when the QD becomes occupied irreversibly escapes out from the QD region in the form of a phonon wind after the interband excitation [7]. The existence of a dynamical reaction of the lattice to the changing of electronic configuration constitutes the most fundamental facet of the irreversibility inherent to the optical response of QDs.

In summary, in a semiconductor QD, the coupling to the continuum of acoustic phonons leads to an optical spectrum that consists in a central ZPL with lateral sidebands. This intrinsic phenomenon hardly depends on the QD material since the interaction with acoustic phonon is only weakly dependent on the polarity of the chemical bound in the crystal elementary cell. At room temperature, the optical spectrum is entirely dominated by the sidebands which spectral extension is of the order of 5 to $10 \mathrm{meV}$ [5]. These values give a lower limit for the QD homogeneous linewidth and thus to width of the spectral gain that one can reach in a semiconductor QD laser with negligible inhomogeneous broadening. In the context of quantum information, the acoustic phonon sidebands do not bring a fundamental limitation to the generation of single photons at room temperature since the antibunching phenomenon is not affected by the fact that the radiative recombination is assisted by the emission or the absorption of acoustic phonons. However, in the prospect of indistinguishable photon generation, any deviation from the radiative limit $\left(T_{2}=2 T_{1}\right)$ reduces the coalescence efficiency of two photons successively generated by the QD [8]. As a matter of fact, the acoustic phonon sidebands severely restrict such a quantum optics scheme at room temperature unless one achieves a control of the acoustic phonon density of states in phononic nanostructures.

In the second part of this paper, we will focus on spectral diffusion which mainly influences the QD optical spectrum in the limit of low temperatures, in contrast to the phenomenon of acoustic phonon sidebands that dominate the spectrum at room temperature. In particular, we will now address the issue of the ZPL width and show the existence of a motional narrowing regime for spectral diffusion.

\section{Spectral diffusion}

At low temperature where the QD optical spectrum is mainly determined by the ZPL, PL experiments in single QDs have revealed the extrinsic influence of the solid matrix that generates fluctuating electric fields and shifts the QD line through the quantum confined Stark effect. This so-called spectral diffusion phenomenon was interpreted as due to carriers randomly trapped in defects, impurities in the QD vicinity $[9,10,11]$. Given the time-resolution imposed by the detector integration time, the emission spectrum can be analyzed by considering two different 
classes of random events. The first one is associated to rare and strongly-shifting processes that result in abrupt jumps of the QD emission energy, i.e. spectral discontinuities in the time-evolution of the PL spectrum. In the case of colloidal QDs or nanocrystals, these events may also inhibit the emission and the corresponding blinking effect [9] is a severe restriction to the realization of efficient single QD-devices based on this type of nanostructures. The second type of events is related to frequent and weakly-shifting processes that lead, within the detector integration time, to a single line with a width that is larger than the radiative limit and that is determined by the statistical distribution of the small energy shifts. We will discuss in the following this second case and in particular show the existence of a motional narrowing regime for spectral diffusion.

\subsection{Zero-phonon linewidth}

In epitaxial QDs, the ZPL often exhibits a Lorentzian profile with a width larger than the radiative broadening, in the $\mu \mathrm{eV}$ range for InGaAs QDs. In PL experiments under non-resonant excitation, the ZPL width ranges from few tens to hundreds of $\mu \mathrm{eV}$ (Fig. 2(b), inset). These values appear to be considerably smaller under resonant excitation of the fundamental interband transition and the limit of radiatively-limited optical spectra is nearly reached either in non-linear four-wave mixing experiments [12] or in linear absorption measurements [13]. Such a dependence of the ZPL width with the excitation energy indicates indeed that spectral diffusion effects can be reduced by a selective excitation of the QD [14]. However, the Lorentzian profile of the ZPL and its width variations with temperature and incident power (inset of Fig. 2(b) and Fig. 3 discussed below) are not consistent with the simple picture of a ZPL given by the statistical distribution of the possible energies of the QD in its fluctuating environment.

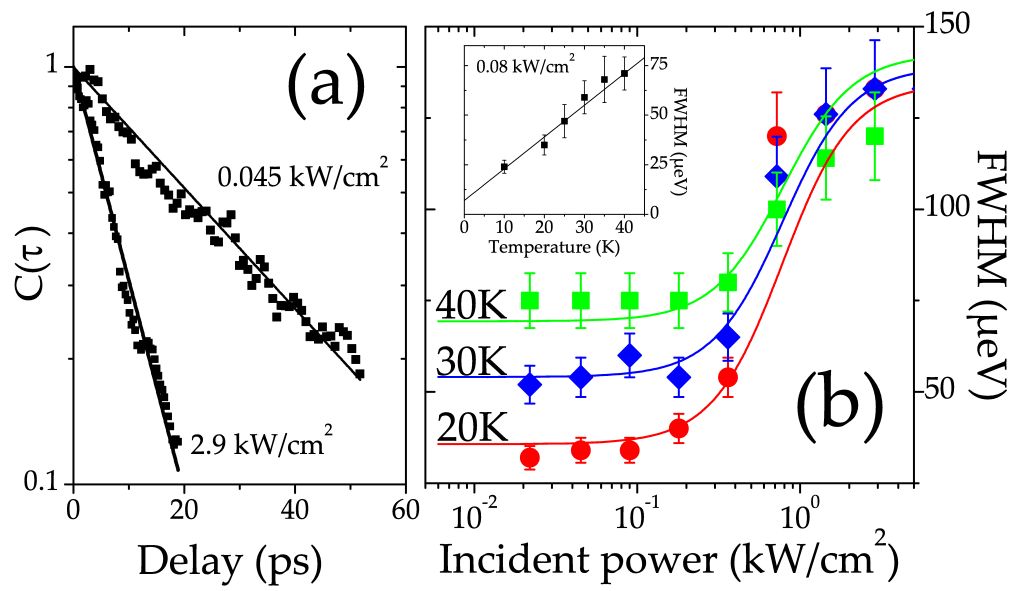

Figure 3. From Ref. [15].(a) Fourier-transform spectroscopy on the PL signal of a single QD at 20K, for two incident powers. (b) Full width at half maximum (FWHM) of the quantum dot line, as a function of incident power for three tempera- tures: $20 \mathrm{~K}$ (red circles), $30 \mathrm{~K}$ (blue diamonds) and $40 \mathrm{~K}$ (green squares). The solid lines (20K in red, $30 \mathrm{~K}$ in blue, $40 \mathrm{~K}$ in green) are calculated within a model taking into account the carrier dynamics in the environment. Inset: Temperature dependence of the FWHM of the quantum dot line for an incident power of $0.08 \mathrm{~kW} . \mathrm{cm}^{-2}$. The solid line is a linear regression of the data with a slope $\alpha_{0} \sim 1.55 \mu \mathrm{eV} . \mathrm{K}^{-1}$.

We display in Fig. 3(b) the full width at half maximum (FWHM) of the PL spectrum of a single InAs/GaAs QD, as a function of incident power for different temperatures [15]. The line-profile remains Lorentzian in the investigated range of incident power and temperature, as can be seen in Fig. 3(a) from the exponential decay of the interferogram contrasts $C(\tau)$ recorded by Fourier-transform spectroscopy [16]. A Lorentzian profile is usually interpreted in terms of homogeneous broadening, which seems in contradiction with the assumption of an extrinsic source of broadening due to spectral diffusion. Moreover, we see in Fig. 3(b) that at 20K, the line-width increases by a factor of 4 over the two decades of incident power, with a pronounced rise around $0.6 \mathrm{~kW} . \mathrm{cm}^{-2}$. At higher temperatures, the variations are qualitatively the same but the line-broadening with incident power is reduced. The line-width increases only by a factor of 2.5 at $30 \mathrm{~K}$, and 1.7 at $40 \mathrm{~K}$. If we analyze the temperature dependence of the ZPL at a given excitation power, we can define the efficiency $\alpha$ of the temperature-induced broadening from the linear regression $\Gamma=\Gamma_{0}+\alpha T$, where the slope $\alpha$ depends on the excitation density. At low incident power $\left(\mathrm{P}<0.2 \mathrm{~kW} . \mathrm{cm}^{-2}\right)$, we find for $\alpha$ a value of $1.55 \pm 0.15 \mu \mathrm{eV} . \mathrm{K}^{-1}$ (Fig. 3(b), inset), i.e. a standard figure compared 
to published data, where $\alpha$ ranges between 0.05 and $4 \mu \mathrm{eV} \cdot \mathrm{K}^{-1}[12,14,17,18]$. At high incident power $(\mathrm{P}>0.2$ $\mathrm{kW} . \mathrm{cm}^{-2}$ ), the higher the excitation density the smaller the ZPL broadening with temperature, and for $\mathrm{P} \gtrsim 2$ $\mathrm{kW} \cdot \mathrm{cm}^{-2}$ the variations lie within our experimental uncertainty.

This example shows that the ZPL broadening can not be characterized by two uncorrelated, additive contributions $\Delta \Gamma(T)$ and $\Delta \Gamma(P)$ that would depend only on temperature and incident power, respectively. The ZPL broadening with temperature is in fact a highly debated issue since no broadening of the ZPL is predicted in the framework of the Huang-Rhys theory developed above, for the interpretation of the lateral sidebands, and which is based on an independent boson model with a linear coupling to acoustic phonons. In a more sophisticated model including a quadratic coupling to acoustic phonons via phonon-assisted transitions to higher QD levels [19], it has been shown that the ZPL width increases linearly with temperature [20]. Nevertheless, the magnitude of this contribution can not quantitatively explain the experimental results $[12,14,17,18]$, and in particular the confinement energy dependence found in Ref. [12]. Phonon damping by scattering at surfaces also results in a linear increase with temperature of the ZPL width and this process was proposed in Ref. [18] to explain the measurements carried out on different mesa sizes. However, the magnitude of this contribution is too small to account for the experimental results in Ref. [17] where single QD spectroscopy is performed without mesas. The dispersion of the data on the temperature dependence of the ZPL width comes from spectral diffusion and we present now a detailed understanding of these observation based on the existence of motional narrowing for spectral diffusion.

\subsection{Motional narrowing}

The influence of spectral diffusion on the optical spectrum of a semiconductor QD can be determined by applying the stochastic Kubo

theory of line-shape and relaxation of a system coupled to a fluctuating reservoir [21]. The random fluctuation over time $\delta E(t)$ of the QD transition energy around a mean value $E_{0}$ are characterized by two quantities. The first one is the modulation amplitude $\Sigma$, which is defined as the standard deviation of the emission energy:

$$
\Sigma=\sqrt{<\delta E(t)^{2}>}
$$

The second one is the correlation time $\tau_{c}$ given by:

$$
\tau_{c}=\frac{1}{\Sigma^{2}} \int_{0}^{\infty} d t<\delta E(t) \delta E(0)>
$$

corresponding to the typical time of exploration of the different reservoir configurations. Assuming that the reservoir fluctuation follow a Gaussian probability law, the Fourier-transform of the intensity spectrum has the analytical expression [21]:

$$
C(t)=\exp \left[-\frac{\Sigma^{2} \tau_{c}^{2}}{\hbar^{2}}\left(\exp \left(-\frac{t}{\tau_{c}}\right)+\frac{t}{\tau_{c}}-1\right)\right]
$$

Depending on the magnitude of the figure of merit $\Sigma \tau_{c} / \hbar$, the optical spectrum is in fact either inhomogeneously broadened with a Gaussian lineshape, or homogeneously broadened with a Lorentzian lineshape. In the limit of $\Sigma \tau_{c} / \hbar \gg 1$ (so-called slow modulation regime), the characteristic function $C(t)$ has a Gaussian decay $\left(C(t) \sim \exp \left(-\Sigma^{2} t^{2} / 2 \hbar^{2}\right)\right)$ : the spectrum reflects directly the statistical distribution of the emission energies, and the line-shape is Gaussian with a FWHM given by $2 \sqrt{2 \ln 2} \Sigma$. In the opposite limit where $\Sigma \tau_{c} / \hbar \ll 1$ (fast modulation regime), a motional narrowing effect gives rise to an exponential decoherence $\left(C(t) \sim \exp \left(-\Sigma^{2} \tau_{c} t / \hbar^{2}\right)\right)$ with a decay time $T_{2}=\hbar^{2} / \Sigma^{2} \tau_{c}$ : the fluctuation is smoothed out by the fast modifications of the reservoir configuration, and the line-shape becomes Lorentzian with a FWHM given by $2 \Sigma^{2} \tau_{c} / \hbar$.

The fluctuating environment can be described in a simple model where $N$ uncorrelated, identical traps individually induce a Stark shift $\Delta$ of the QD emission line. The carrier dynamics in the traps is characterized by a capture time $\tau_{\downarrow}$ and an escape time $\tau_{\uparrow}$. The correlation time $\tau_{c}$ and the spectral modulation amplitude $\Sigma$ are then given by the expressions $1 / \tau_{c}=1 / \tau_{\uparrow}+1 / \tau_{\downarrow}$ and $\Sigma=2 \Sigma_{s} /\left(\sqrt{\tau_{\uparrow} / \tau_{\downarrow}}+\sqrt{\tau_{\downarrow} / \tau_{\uparrow}}\right)$, respectively, where the maximum value $\Sigma_{s}$ of the modulation amplitude is reached for $\tau_{\downarrow} \sim \tau_{\uparrow}$ and is equal to $\sqrt{N} \Delta / 2$ [22].

In Ref. [22], we showed that the motional narrowing regime strikingly occurs when decreasing the incident power or the temperature in contrast to the text-book phenomenology described in nuclear magnetic resonance. An example is presented in Fig. 4 where a smooth transition from the fast-modulation limit to the slow-modulation one is observed on increasing incident power. We display there the measured (squares) interference contrast $C(t)$ for the emission spectrum of a single InAs/GaAs QD at 10K, on semi-logarithmic plots, for three different incident powers: 0.18 (a), $0.72(\mathrm{~b})$, and $2.88 \mathrm{~kW} \cdot \mathrm{cm}^{-2}$ (c). A comparison between the experimental data and the general 


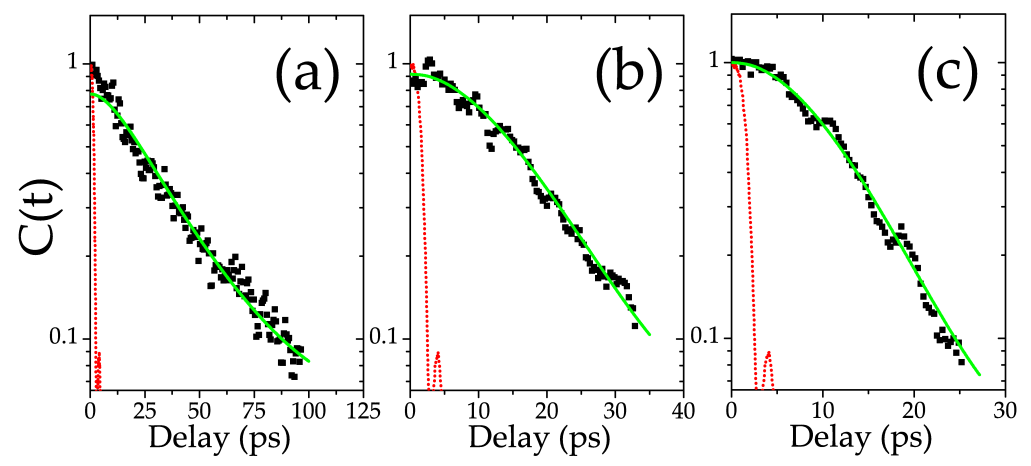

Figure 4. From Ref. [22].Interferogram contrast C(t) of the photoluminescence signal of a single InAs/GaAs QD at 10K, on semi-logarithmic plots, for three different incident powers: 0.18 (a), 0.72 (b), and $2.88 \mathrm{~kW} . \mathrm{cm}^{-2}$ (c). Data (squares), system response function (dotted line), theoretical fits (solid line) obtained by the convolution of the system response function with Eq. (3), with $\Sigma \tau_{c} / \hbar \sim$ 0.6 (a), 1.05 (b), and 1.35 (c), are plotted as a function of the delay t.

expression of $\mathrm{C}(\mathrm{t})$ given in Eq. (8) allows us to quantify the transition from the exponential to the Gaussian relaxation. The calculated fits are obtained by convoluting Eq. (8) with the system response function (dotted lines in Fig. 4). An excellent agreement is reached by taking a constant correlation time $\tau_{c} \sim 10$ ps and increasing values of $\Sigma \tau_{c} / \hbar$ of 0.6 in (a), 1.05 in (b), and 1.35 in (c). The transition from the fast modulation limit to the slow modulation occurs when $\Sigma \tau_{c} / \hbar$ becomes larger than one. This unconventional manifestation of motional narrowing is quantitatively interpreted and the asymmetry of the capture and escape mechanisms appears to be the fundamental reason why motional narrowing strikingly occurs in the limit of low temperature and low incident power [22]. In the limit where $\tau_{\downarrow} / \tau_{\uparrow} \ll 1$ corresponding to an escape process much less efficient than the capture one, the modulation amplitude $\Sigma$ increases linearly with $\sqrt{\tau_{\downarrow} / \tau_{\uparrow}}$ and $\tau_{c} \sim \tau_{\downarrow}$. As a matter of fact, when $\tau_{\uparrow}$ decreases with temperature, the correlation time is merely constant, the modulation amplitude increases so that $\Sigma \tau_{c} \hbar$ is small at low temperature thus explaining the Lorentzian profile. In NMR, the situation is the opposite one: $\Sigma$ is constant, $\tau_{c}$ decreases with temperature so that $\Sigma \tau_{c} \hbar$ is small at high temperature with a Lorentzian profile at high temperature.

The fits displayed in solid line in Fig. 3(b) are calculated within the same model where the capture and escape times of the carriers in the traps around the QD depend on incident power and temperature because of Auger and phonon-assisted processes, respectively. The excellent agreement between experimental data and calculations show that the variations of the ZPL width are given by the activation of spectral diffusion with incident power and temperature. The reduction of the temperature-broadening efficiency $\alpha$ on increasing incident power is not specific to spectral diffusion but it comes for this particular QD from the interplay between Auger-assisted and phonon-assisted processes involved in the carrier escape from the traps in the QD environment [15].

These results show that the carrier dynamics in the QD environment provides a significant contribution to the temperature-induced dephasing in the low temperature limit $(\mathrm{T}<50 \mathrm{~K})$. In contrast to the model proposed in Ref. $[18,20]$, the temperature-dependence of the ZPL width comes from the interaction of acoustic phonons with carriers outside the QD. This purely extrinsic contribution suggests the picture of the decoherence dynamics in QDs as a probe of the carrier dynamics in the QD environment and provides a simple interpretation to the wide dispersion of the published experimental data on the ZPL broadening $[12,14,17,18]$.

In summary, the QD matrix contributes to the decoherence dynamics through the extrinsic process of spectral diffusion and we have shown the existence of two different fluctuation regimes: the slow modulation one where the QD optical spectrum directly reflects the statistical distribution of the QD emission energy, and the fast modulation one, also called motional narrowing regime, where the QD line is a Lorentzian with a width smaller than the standard deviation of the QD energy. The motional narrowing strikingly occurs in the limit of low temperature or low incident power, in contrast to the text book example of nuclear magnetic resonance. The motional narrowing regime for spectral diffusion explains the observation in single QDs of sharp Lorentzian lines with a width larger than the radiative broadening. Moreover, we stress that the carrier dynamics in the QD environment provides a significant contribution to the temperature-induced dephasing in the low temperature limit, and accounts for the linear temperature dependence of the ZPL width at low temperatures. 


\section{Conclusion}

We have shown that the PL spectrum of epitaxial QDs very often display a series of deviations from a simply radiatively broadened Lorentzian line. These deviations are related to different physical processes, which conspire to enhance the dephasing of the optical response of such objects, and are ultimately inherent to the existence of a nanometric localization of carriers in a solid-state matrix. Firstly, the non-perturbative reaction of the lattice to the interband transition explains the observed sidebands in the PL spectrum. Even though the physical explanation is not new (the coupling by deformation potential and the Huang-Rhys theory date from the 50s), its observation in nanostructures has been important in two ways : (i) to corroborate simple electronic level and phonon modes calculation often used to describe the properties of QDs, and, more importantly, (ii) to emphasize the existence of an intrinsic (thus unavoidable) dephasing for the optical transitions of QDs. Secondly, the origin of the ZPL width has been for many years a subject of controversy. The recent detailed studies reviewed in this paper paves the way for a conclusive answer to this issue, while allowing revealing an original phenomenology in terms of an unconventional motional narrowing effect involving the QD and its nearby environment.

The works reported in this review definitely rank the non-perturbative coupling to acoustical phonons and the effects of a fluctuating environment as two unavoidable ingredients in any model aiming describing the coherent optical response of QDs. However, further works are desirable to more clearly precise the origin of the latter. In fact, our simple model in terms of charging/decharging processes involving a series of trapping centers captures one essential part of the fluctuating phenomenon, but detailed investigations of the nature and spatial distribution of such defects in actual samples are still missing. Also, we have seen that phonon effects concern both low confining interface-related QDs and strongly confining epitaxial QDs. This may have important additional implications, which could motivate future works. For instance, one could explore the possibility of a long-range relationship between optical processes in different QDs, mediated by acoustical phonons : the phonon wind generated by the radiative recombination in one QD may act as a noise source for the recombination of pairs in a neighbor QD, even though their direct (electrostatic) coupling is negligible. For an ensemble of dots this process ought to be accounted for in a self-consistent way. Moreover, trapping processes involving low-confining defects should also in principle be accompanied by dynamical lattice reaction, which would then also act as a new source of environment fluctuation. In this context, interband recombination in a QD is an optical process in an environment that is noisy in both electrostatic and acoustical senses.

\section{Acknowledgements}

We gratefully acknowledge A. Berthelot, I. Favero, C. Voisin, Ph. Roussignol, G. Bastard, and J. M. Gérard for stimulating discussions.

\section{References}

[1] L. Besombes, K. Kheng, L. Marsal and H. Mariette, Phys. Rev. B 63, 155307 (2001).

[2] I. Favero, G. Cassabois, R. Ferreira, D. Darson, C. Voisin, J. Tignon, C. Delalande, G. Bastard, Ph. Roussignol, and J. M. Gérard, Phys. Rev. B 68, 233301 (2003).

[3] E. Peter, J. Hours, P. Senellart, A. Vasanelli, A. Cavanna, J. Bloch, and J. M. Gérard, Phys. Rev. B 69, 041307 (2004)

[4] P. Borri, W. Langbein, S. Schneider, U. Woggon, R. L. Sellin, D. Ouyang, and D. Bimberg, Phys. Rev. Lett. 87, 157401 (2001).

[5] M. Bayer and A. Forchel, Phys. Rev. B 65, 41308 (2002).

[6] C. B. Duke and G. D. Mahan, Phys. Rev. 139, A1965 (1965).

[7] A. Vagov, V. M. Axt and T. Kuhn, Phys. Rev. B 66, 165312 (2002).

[8] J. Bylander, I. Robert-Philip, and I. Abram, Eur. Phys. J. D 22, 295-301 (2003).

[9] S. A. Empedocles, D. J. Norris, and M. G. Bawendi, Phys. Rev. Lett. 77, 3873 (1996).

[10] H. D. Robinson and B. B. Goldberg, Phys. Rev. B 61, R5086 (2000).

[11] V. Türck, S. Rodt, O. Stier, R. Heitz, R. Engelhardt, U. W. Pohl, D. Bimberg, and R. Steingrüber, Phys. Rev. B 61, 9944 (2000).

[12] P. Borri, W. Langbein, U. Woggon, V. Stavarache, D. Reuter, and A. D. Wieck, Phys. Rev. B 71, 115328 (2005).

[13] A. Högele, S. Seidl, M. Kroner, K. Karrai, R. J. Warburton, B. D. Gerardot, and P. M. Petroff, Phys. Rev. Lett. 93, 217401 (2004).

[14] C. Kammerer, C. Voisin, G. Cassabois, C. Delalande, Ph. Roussignol, F. Klopf, J. P. Reithmaier, A. Forchel, and J. M. Gérard, Phys. Rev. B 66, 041306 (2002).

[15] I. Favero, A. Berthelot, G. Cassabois, C. Voisin, C. Delalande, Ph. Roussignol, R. Ferreira, and J. M. Gérard, Phys. Rev. B 75, 073308 (2007) 
[16] C. Kammerer, G. Cassabois, C. Voisin, M. Perrin, C. Delalande, Ph. Roussignol, and J. M. Gérard, Appl. Phys. Lett. 81, 2737 (2002).

[17] B. Urbaszek, E. J. McGhee, M. Krüger, R. J. Warburton, K. Karrai, T. Amand, B. D. Gerardot, P. M. Petroff, and J. M. Garcia, Phys. Rev. B 69, 035304 (2004).

[18] G. Ortner, D. R. Yakovlev, M. Bayer, S. Rudin, T. L. Reinecke, S. Fafard, Z. Wasilewski, and A. Forchel, Phys. Rev. B 70, 201301 (2004).

[19] T. Takagahara, Phys. Rev. B 60, 2638 (1999).

[20] E. A. Muljarov and R. Zimmermann, Phys. Rev. Lett. 93, 237401 (2004)

[21] R. Kubo, A stochastic theory of line-shape and relaxation, in Fluctuation, Relaxation and Resonance in Magnetic Systems (ed D. Ter Haar) 23-68 (Oliver and Boyd, Edinburgh, 1962).

[22] A. Berthelot, I. Favero, G. Cassabois, C. Voisin, C. Delalande, Ph. Roussignol, R. Ferreira, and J. M. Gérard, Nature Phys. 2, $759(2006)$ 